\title{
Takotsubo-Kardiomyopathie
}

\section{Eine Folge der arteriellen Hypertonie?}

Die Symptomatik, das EKG und die Serummarker sprechen für einen Myokardinfarkt, doch die Koronarangiografie zeigt keine relevanten Gefäßveränderungen ( $M M W$ Nr. 1-2/2007, S. 41). Eine Leserzuschrift mit einem möglicherweise wichtigen Hinweis zum Takotsubosyndrom:

- Die Takotsubo-Kardiomyopathie wird in der Fachliteratur sehr intensiv diskutiert. Wir wollen eine für die Patienten wichtige Bemerkung anbringen und unsere Erfahrungen mit dieser Kardiomyopathie in einer kardiologischen Gemeinschaftspraxis mitteilen:

Wir haben zwei Patientinnen mit einer mutmaßlichen Takotsubo-Kardiomyopathie behandelt. Beide Patientinnen hatten eine arterielle Hypertonie mit hypertensiver Herzerkrankung,

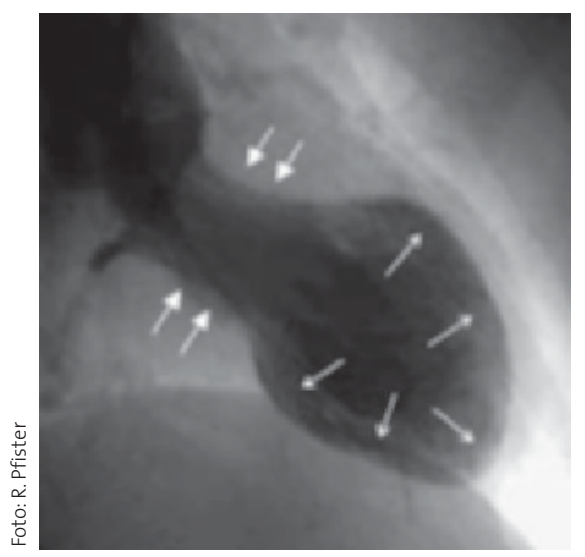

Der linke Ventrikel sieht aus wie ein japanisches Tongefäß (Takotsubo).

die nicht bekannt war oder unterschätzt wurde. Bei der ersten Patientin wurde der Bluthochdruck insuffizient behandelt. Die Hochdrucktherapie wurde nach dem Ereignis in unserer
Praxis verdreifacht! Bei der zweiten Patientin war kein Bluthochdruck bekannt. Im Verlauf benötigte sie eine zweifach antihypertensive BlutdruckKombinationstherapie.

Auch die Patientinnen 1 bis 3 hatten in Ihrer Kasuistik eine arterielle Hypertonie. Wir denken, dass die arterielle Hypertonie und hypertensive Herzerkrankung eine entscheidende Rolle bei der Entwicklung einer Takotsubo-Kardiomyopathie spielt und dies erhebliche therapeutische Konsequenzen für die Patienten hat.

Unsere Empfehlung: Jeder Patient mit Takotsubo-Kardiomyopathie sollte eine strengste Blutdrucküberwachung und eine 24-Stunden-Blutdruckmessung haben!

\footnotetext{
- Dr. med. Johann L. Fischer,

Kardiologische Gemeinschaftspraxis, Putzbrunner Straße 1, D-81737 München
}

\section{Auswüchse des Schönheitswahns}

\section{Männer mit dem Wunsch nach Mastektomie}

\section{Erfolge und Fehlleistungen der Schönheitschirurgie waren das The- ma unseres Reports in MMW Nr. 27-28/2007, S. 10. Ein Leser macht auf eine häufige psychiatrische Störung im Zusammenhang mit dem Schön- heitswahn aufmerksam:}

- Aus der versicherungsmedizinischen Begutachtung, die die Abgrenzung von medizinisch notwendigen plastischchirurgischen Eingriffen zu Schönheitsoperationen zum Ziel hat, ergibt sich nicht selten der Eindruck einer Körperschemastörung im Sinne einer Dysmorphophobie (körperdysmorphe Störung). Die betroffenen Patienten/ Patientinnen sind der unkorrigierbaren Überzeugung, dass sie hässlich oder gar entstellt sind, ohne dass dies bei objektiver Betrachtung der Fall ist. Am häufigsten betroffen sind dabei Nase, Ohren und Busen. Ist dann das entsprechende Körperteil operiert, wird das nächste ins Visier genommen.

Dysmorphophobie betrifft auch Männer. Gerade in den letzten Monaten sind mir mehrere Fälle von angeblicher Gynäkomastie untergekommen, in denen die Männer eine Mastektomie wünschten.

\footnotetext{
- Dr. med. Rainer Hakimi, Facharzt für Allgemeinmedizin, Psychotherapie, Betriebsmedizin, Schickhardtstraße 33, D-70199 Stuttgart
}

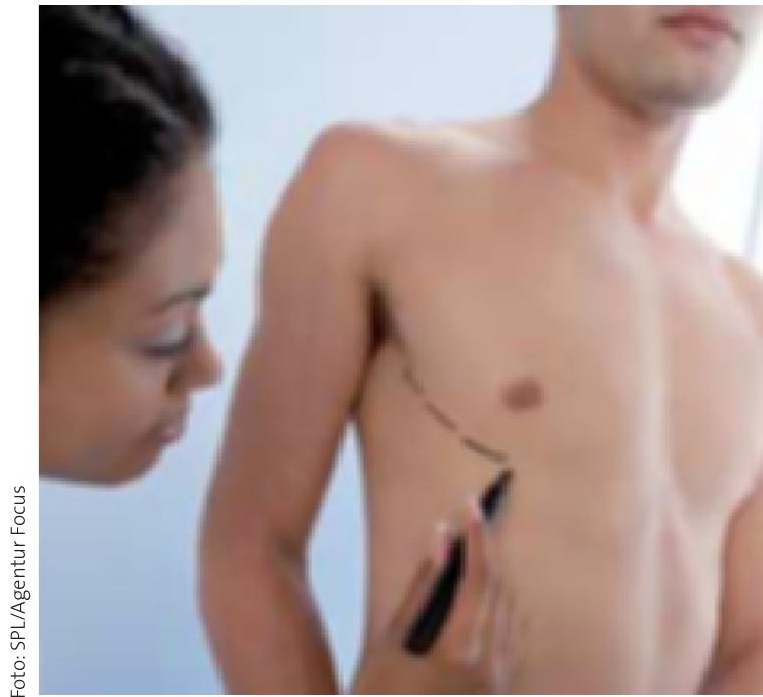

Körpertuning für den Mann (hier: Vorbereitung für eine Pectoralisplastik). 